\title{
How have our clocks evolved? Adaptive and demographic history of the out-of-African dispersal told by the genetic markers of morning-evening preference
}

\author{
A.A. Putilov ${ }^{1 *}$, V.B. Dorokhov ${ }^{2}$, A.N. Puchkova ${ }^{2,3}$, D.S. Sveshnikov ${ }^{4}$, \\ M.G. Poluektov ${ }^{5}$, O.G. Donskaya ${ }^{1}$, L.P. Zakharenko ${ }^{6}$ \\ ${ }^{1}$ Institute of Molecular Biology and Biophysics, Novosibirsk, Russia \\ ${ }^{2}$ Institute of Higher Nervous Activity and Neurophysiology RAS, Moscow, Russia \\ ${ }^{3}$ Pushkin State Russian Language Institute, Moscow, Russia \\ ${ }^{4}$ Peoples' Friendship University of Russia, Moscow, Russia \\ ${ }^{5}$ I.M. Sechenov $1^{\text {st }}$ Moscow State Medical University, Moscow, Russia \\ ${ }^{6}$ Institute of Cytology and Genetics SB RAS, Novosibirsk, Russia \\ *e-mail:putilov@ngs.ru
}

Key words: SNP, 1000 Genomes Project, latitudinal cline, morning-evening preference, polygenic selection, skin pigmentation, Neanderthal's genome, migration out of Africa

Motivation and Aim: The natural cycles of night and day, and their length, remain stable in near-equatorial African regions but they vary with latitude and season in Eurasia. Therefore, this new environmental factor seemed to shape the adaptation of the circadian rhythms of Eurasians after the out-of-African dispersal of their African ancestors [1]. We tried to identify the genetic-based signatures of this latitude-dependent adaptation.

Methods and Algorithms: Geographic variation in allele frequencies of more than 28 hundred genetic variants was analyzed using data from 5 African and 11 Eurasian populations sampled for the 1000 Genomes Project Phase 3 (1594 individuals).

Results: As we expected, the genetic signatures of latitude-dependent polygenic selection were found more frequently within non-coding DNA regions associated with morningevening preference in 4 genome-wide association studies (GWASs). These signatures were identified less frequently among polymorphisms hinted by GWASs of other traits/ diseases and among polymorphisms sampled from pseudogenes and from proteincoding regions in either circadian clock genes or reference genes. The frequencies were also high for loci associated with those traits that, like morning-evening preference, were shaped by latitude-dependent adaptations (e.g. skin pigmentation). Some of these genetic variants were located within the introgressions of the Neanderthal's genome into the genomes of Eurasians.

Conclusion: A promising approach to prioritization of genetic markers of morningevening preference would be to aim future candidate gene studies on examination of the strength of association of this trait with a set of loci harboring latitude-dependent adaptations.

Availability: The statistics on the analyzed loci are available from the authors upon request.

Acknowledgements: AAP and OGD were supported by a grant from the Russian Foundation for Basic Research (grant number 16-06-00235-a).

\section{Reference}

1. Putilov A.A. et al. (2018) How have our clocks evolved? Adaptive and demographic history of the outof-African dispersal told by polymorphic loci in circadian genes. Chronobiol Int. 34:(online link: http:// dx.doi.org/10.1080/07420528.2017.1417314) 